\title{
Diversity and functional analysis of luxS genes in Vibrios from marine sponges Mycale laxissima and Ircinia strobilina
}

\author{
Jindong Zan ${ }^{1}$, Clay Fuqua ${ }^{2}$ and Russell T Hill ${ }^{1}$ \\ ${ }^{1}$ Institute of Marine and Environmental Technology, University of Maryland Center for Environmental \\ Science, Baltimore, MD, USA and ${ }^{2}$ Department of Biology, Indiana University, Bloomington, IN, USA
}

\begin{abstract}
Sponges harbor highly diverse and dense microbial communities, providing an environment in which bacterial signaling may be important. Quorum sensing (QS) is a cell density-dependent signaling process that bacteria employ to coordinate and regulate their gene expression. Previous studies have found that bacteria isolated from sponges are able to produce acyl-homoserine lactones (AHLs), an important class of QS molecules found in proteobacteria. Autoinducer-2 (Al-2) is a second class of QS molecule, and is considered to be an interspecies signal. However, Al-2 signaling has not been reported in sponge bacterial symbionts. In this study, degenerate primers were designed based on known Vibrio luxS sequences to amplify the luxS genes encoding Al-2 synthases of several Vibrio isolates from marine sponges Mycale laxissima and Ircinia strobilina. All the vibrios isolated from these two sponges had luxS genes and were able to produce signals with Al-2 activity as detected using a biological reporter. A novel group of luxS sequences was found, thus extending the known diversity of luxS genes. One isolate was chosen for further analysis of its luxS gene by expression of the gene in Escherichia coli $\mathrm{DH} 5 \alpha$ and by characterization of the profile of Al-2 activity. This work provides the first information about luxS genes and Al-2 activity in sponge-associated bacterial communities.

The ISME Journal (2011) 5, 1505-1516; doi:10.1038/ismej.2011.31; published online 31 March 2011
\end{abstract}

Subject Category: microbe-microbe and microbe-host interactions

Keywords: sponges; symbionts; quorum sensing; acyl-homoserine lactone; autoinducer-2; Vibrio

\section{Introduction}

Sponges (phylum Porifera) are one of the most primitive multicellular animals and are sessile, filter-feeders. Sponges can form close associations with microbes that in some cases comprise up to $60 \%$ of the biomass of the sponges (Vacelet, 1975; Vacelet and Donadey, 1977; Wilkinson, 1978; Hentschel et al., 2006). Molecular techniques have revealed that these bacterial communities are remarkably diverse and include many novel bacteria (Taylor et al., 2007; Mohamed et al., 2008c; Webster and Blackall, 2009). Symbiotic relationships between sponges and microorganisms are considered to contribute to the health and nutrition of sponges (Mohamed et al., 2010). Many studies have shown the metabolic phenotypes of sponge-associated microbes (Taylor et al., 2007; Mohamed et al., 2008d, 2010), although little evidence exists confirming the contribution of symbiotic microbes to

Correspondence: RT Hill, Institute of Marine and Environmental Technology, University of Maryland Center for Environmental Science, Columbus Center Suite 236, 701 East Pratt Street, Baltimore, MD 21202, USA.

E-mail: hill@umces.edu

Received 5 July 2010; revised 1 February 2011; accepted 20 February 2011; published online 31 March 2011 sponge well-being or survival (Webster and Blackall, 2009). Exceptions are the translocation of photosynthate from cyanobacteria to the host sponge (Wilkinson, 1979) and a decrease in health status of sponges associated with loss of cyanobacteria (Thacker, 2005).

Bacteria are known to effectively communicate with each other (Hooshangi and Bentley, 2008). One of the mechanisms for communication is termed quorum sensing (QS), in which bacteria monitor and respond to their own population density. In this process, diffusible signal molecules, sometimes called autoinducers, are produced at basal levels. The signals can accumulate proportionally to population density, and at critical threshold concentration, the signal binds to receptor proteins, which trigger expression of a specific spectrum of genes and phenotypes (Fuqua et al., 1994). Bacterial processes such as biofilm formation, bioluminescence, motility, virulence factor secretion, antibiotic production, sporulation and competence for DNA uptake can be controlled by QS systems ( $\mathrm{Ng}$ and Bassler, 2009).

In Proteobacteria, acyl-homoserine lactones (AHLs) are a major type of molecular cue for QS, and were originally described as autoinducers. Bacteria that are able to produce AHLs have been 
isolated from marine sponges (Taylor et al., 2004; Mohamed et al., 2008a). Sponges harbor a higher proportion of AHL producers in their culturable bacterial communities compared with that of surrounding seawater (Mohamed et al., 2008a). AHL regulatory systems among these bacteria may have roles during bacterial colonization of the sponges or in controlling activities within the sponge symbiont community (Cicirelli, Hill and Fuqua, in preparation). They may also provide one means by which sponges could interact or interfere with bacteria (Taylor et al., 2007).

Autoinducer-2 (AI-2) is another well-known molecular cue and has been extensively analyzed in Vibrio harveyi and Vibrio cholerae, where it is involved in regulation of bioluminescence and virulence-associated traits (Miller et al., 2002; Lenz et al., 2004; Henke and Bassler, 2004a,b). The activated methyl cycle is a crucial metabolic pathway to recycle homocysteine from the major methyl donor $S$-adenosyl methionine. LuxS, an $S$-ribosylhomocysteinase, catalyzes part of the cycle and functions to convert $S$-ribosylhomocysteine to homocysteine; meanwhile, it can also, as a side reaction, synthesize 4,5-dihydroxy-2,3-pentanedione, the precursor of AI-2. 4,5-Dihydroxy-2,3pentanedione can spontaneously give rise to several furanone derivatives, collectively referred to as AI-2. The dual roles of luxS in metabolism and in AI-2 formation have led to controversy regarding its function in QS (Doherty et al., 2006; Rezzonico and Duffy, 2008). $V$. harveyi produces a third type of autoinducer CAI-1, an alpha-hydroxyketone (Tiaden et al., 2010), in addition to the AHL autoinducer (3-OH-C4-HSL) and AI-2. The three different types of autoinducers in $V$. harveyi bind to their respective membrane-bound receptors and converge on a central signal transduction pathway to regulate gene expressions (Ng and Bassler, 2009).

Thus far, no studies have been performed on the AI-2 systems among sponge-associated bacteria. During the process of isolating bacteria from the sponges Mycale laxissima and Ircinia strobilina, we obtained many Vibrio strains. We therefore focused on this investigation of the presence of luxS genes in sponge-associated bacteria on Vibrio spp. because of their high incidence in the sponges. Degenerate primers of luxS based on known Vibrio luxS sequences were designed and applied to all the Vibrio isolates. The AI-2 activity of all the Vibrio isolates was tested and a novel luxS sequence was cloned and analyzed further. The same set of primers was used to PCR-amplify the luxS genes directly from sponge bacterial communities.

\section{Materials and methods}

\section{Sample collection}

Three individuals of each of $M$. laxissima and I. strobilina were collected by SCUBA at Conch
Reef, Key Largo, FL, USA in late October 2008 at a depth of $c a 20 \mathrm{~m}$. The water salinity was measured to be 36 p.p.t. using a portable refractometer (Fisher Scientific, Pittsburgh, PA, USA) at a water temperature ca $28^{\circ} \mathrm{C}$. Sponge samples were transported to the laboratory within $1 \mathrm{~h}$ of collection and sponges were rinsed three times with sterile artificial seawater for bacterial isolation and frozen at $-20^{\circ} \mathrm{C}$ for later DNA extraction. Water samples were collected within $1 \mathrm{~m}$ of the sponges at a depth of $c a 20 \mathrm{~m}$ in a sterile 20-l container. Approximately $6 \mathrm{l}$ of water was filtered through each of three 0.22 um pore-size Sterivex filters (Millipore, Billerica, MA, USA). The filters for extraction of DNA from bacteria in the water samples were flushed with 1X SET buffer (0.1 M NaCl, $10 \mathrm{~mm}$ Tris-Hcl, pH 8.0 $1 \mathrm{~mm}$ EDTA) as described by Somerville et al. (1989) and stored at $-20^{\circ} \mathrm{C}$.

\section{Bacterial isolation and enumeration}

Sponges were rinsed with sterile artificial seawater to remove transiently associated bacteria and then processed for isolation of culturable bacteria. Sponge tissue $\left(1 \mathrm{~cm}^{3}\right)$ was ground in artificial seawater using a sterile mortal and pestle and 10-fold serial dilutions were plated on Difco Marine Agar 2216 (BD Biosciences, Franklin Lakes, NJ, USA). Plates were incubated at $30^{\circ} \mathrm{C}$ for 1 week at which time bacterial colonies were counted on plates with suitable dilutions (30-300 colonies). Approximately 50 colonies were selected for identification by $16 \mathrm{~S}$ ribosomal RNA (rRNA) gene sequencing (below) to determine the proportion of vibrios in the culturable community. Serial dilutions of water samples were processed similarly for bacterial isolation. Total bacterial counts were determined using methods described by Mohamed et al. (2008d).

\section{Identification of bacterial isolates by $16 S$ rRNA gene} sequence analysis

A single colony of each isolate was transferred to $15 \mathrm{ml}$ of Marine Broth 2216 and incubated at $30^{\circ} \mathrm{C}$ for 24-48 h. Bacterial genomic DNA was extracted from isolates using the UltraClean microbial kit (MoBio Laboratories, Carlsbad, CA, USA). Representatives of each morphotype based on careful observation of colony appearance were subcultured and cryopreserved at $-80{ }^{\circ} \mathrm{C}$ in Marine Broth 2216 supplemented with $30 \%$ glycerol. Almost fulllength 16S rRNA gene fragments were PCR-amplified using universal primers $27 \mathrm{~F}$ and $1492 \mathrm{R}$ as described by Enticknap et al. (2006). PCR products were sequenced using an ABI PRISM 3130xl genetic analyzer (Applied Biosystems, Foster City, CA, USA) and primers 27F and1492R. Sequences were assembled using online software CAP3 (http:// pbil.univ-lyon1.fr/cap3.php) with a manual check. Chimeric sequences were identified by using the 
CHECK_CHIMERA program of the Ribosomal Database Project (Maidak et al., 2001) and the sequences were analyzed initially by using the BLASTn tool at the National Center for Biotechnology Information website (NCBI).

Extraction of genomic DNA from sponges and surrounding water samples

Freeze-dried sponge tissue $\left(1 \mathrm{~cm}^{3}\right)$ was ground using a sterile mortar and pestle. Total genomic DNA was extracted by a published method (Pitcher et al., 1989), modified for sponge tissues (Enticknap et al., 2006; Mohamed et al., 2008d). DNA was extracted from the filters obtained from surrounding seawater samples as described by Somerville et al. (1989).

\section{Amplification of partial luxS gene sequences from Vibrio isolates}

On the basis of sequences of known Vibrio luxS genes downloaded from Genbank, degenerate primers were designed for amplification of luxS gene fragments using online primer design software consensus-degenerate hybrid oligonucleotide primers (Rose et al., 2003). The primers were designated VluxsF (5'-TGCTGGACTCCTTCACCGTNGAYCAYAC-3') and VluxsR (5'-TGCATGGCGGCGGTNCCRCAYTGTT-3'). These primers were designed based on an alignment of all available Vibrio luxS gene sequences. PCR mixtures consisted of $50 \mu \mathrm{l}$ containing two units Platinum Taq polymerase (Invitrogen Life Technologies, Carlsbad, CA, USA), $1 \times$ PCR Buffer, $2 \mathrm{mM}$ $\mathrm{MgCl}_{2}, 200 \mathrm{mM}$ dNTPS (Fermentas, Glen Burnie, $\mathrm{MD}$, USA), $0.2 \mu \mathrm{M}$ each primer and 10-20 ng of genomic DNA or distilled water as a negative control. PCR cycling conditions for Vibrio luxS gene amplification consisted of $94^{\circ} \mathrm{C}$ for $5 \mathrm{~min}$ followed by 30 cycles of $1 \mathrm{~min}$ at $94{ }^{\circ} \mathrm{C}, 1 \mathrm{~min}$ at $52^{\circ} \mathrm{C}$, and $1 \mathrm{~min}$ at $72^{\circ} \mathrm{C}$. A final $10 \mathrm{~min}$ extension step was done at $72{ }^{\circ} \mathrm{C}$. PCR reactions were performed in a PTC-200 cycling system (Bio-Rad, Hercules, CA, USA). The PCR products with expected size $\sim 400 \mathrm{bp}$ were purified using QIAquick gel extraction kit (Qiagen, Valencia, CA, USA) and sequenced as described above for $16 \mathrm{~S}$ rRNA gene fragments using primer VluxsF.

\section{Measurement of AI-2 activity}

AI-2 activity was detected by using the reporter strain $V$. harveyi TL-26, in which all three signaling pathways are disrupted. Receptor genes for AHL and CAI-1 are mutated as well as the luxS gene. Thus, TL-26 can respond only to exogenously added AI-2 (not AHLs or CAI) and cannot produce AI-2 (Long and Bassler; personal communication). All the Vibrio isolates and $V$. harveyi TL-26 were grown overnight in Luria-Bertani medium with $2 \% \mathrm{NaCl}$. Culture supernatants of test strains were filter sterilized $(0.22 \mu \mathrm{m})$ and $60 \mu \mathrm{l}$ of sterile supernatant was added to $140 \mu \mathrm{l} \mathrm{AB}$ medium (Greenberg et al.,
1979) containing $V$. harveyi TL-26 (inoculated 1:5000 from an overnight culture). Agrobacterium tumefaciens KYC55, which does not produce AI-2 (Zhu et al., 2003), was used as a control to test if this assay is specific. Vibrio mimicus ATCC 33653 was used as a positive control. The culture was incubated at $30^{\circ} \mathrm{C}$ with shaking at 180 r.p.m. for $16 \mathrm{~h}$ and light production was measured by using a FLUOstar OPTIMA fluorescence microplate reader (BMGlabtech, Cary, NC, USA). The induction of luminescence by each tested supernatant was expressed relative to that in a negative control comprising sterile Luria-Bertani medium instead of culture supernatant.

AI-2 activity of JZ08IS72, one Vibrio isolate from the sponge I. strobilina, at different growth phases was measured. Briefly, a culture of JZ08IS72 grown in Luria-Bertani medium with $2 \% \mathrm{NaCl}$, incubated at $30^{\circ} \mathrm{C}$ and shaken at 200 r.p.m. was sampled at several different points over a continuous period of $30 \mathrm{~h}$. AI-2 activity was measured as described above.

Full-length cloning of luxS gene from isolate JZ08IS72 The full-length luxS gene (hereafter referred to here as $\operatorname{lux} S_{72}$ ) of isolate JZ08IS72 from the sponge I. strobilina was cloned using a GenomeWalker Universal Kit according to the manufacturer's instructions (Clontech Laboratories, Inc., Mountain View, CA, USA). Briefly, genomic DNA of JZ08IS72 was extracted using a CTAB method (Murray and Thompson, 1980). The DNA was digested with restriction enzymes provided by the kit and ligated to Genome Walker adaptors to construct a genomic library. The library was screened by $\operatorname{lux} S_{72}$ specific primers using a nested PCR method. The primer used for the first round PCR amplification of the $5^{\prime}$ end of the gene was luxsR1 (5'-GGTATGGATACCCT TTTCAGAAAGCAG-3' ${ }^{\prime}$ and for the second round was luxsR2 (5'-CTGAGTAAAGCGAAGGTCAAACA CAGT $\left.-3^{\prime}\right)$. The primer used for the first round PCR amplification for the $3^{\prime}$ end was luxF1 ( $5^{\prime}$-CGTTGAG ATCATCGACATCTCTCCTAT-3') and for second round was luxF2 (5'- TACATGAGTCTGATCGGTAC ACCTACT- $3^{\prime}$ ). The primers targeting the adaptor sequences were provided by the kit. All the PCR conditions were the same as recommended by the manufacturer. The PCR products were gel purified and sequenced. The sequences were aligned using Clustal X 2.0.12 (http://www.clustal.org/) and assembled using online software CAP3 (http://pbil. univ-lyon1.fr/cap3.php) and also checked manually. The assembled sequences were further analyzed by BLAST.

Heterologous expression of $\operatorname{luxS}_{72}$ in Escherichia coli DH5 $\alpha$

The coding sequence of $\operatorname{lux} S_{72}$ was PCR amplified using forward primer 5'-GCCCTGCAGCTGAAAC $A G$ GAAACAGCTATGCCTTTACTAGATAGC-3' (the $\overline{P_{s t I}}$ 
recognition site is underlined and the ShineDalgarno sequence from lac $Z$ is both underlined and in italics) and reverse primer $5^{\prime}$-GGCCTCGAGT TACACCTTGAGGG-3' (the XhoI recognition site is underlined). The PCR product was cloned into pCR4-TOPO vector (Invitrogen Life Technologies) and then subcloned into expression vector pSRKGm (Khan et al., 2008), named pSRKGm $\operatorname{lux} S_{72}$ and subsequently introduced into $E$. coli $\mathrm{DH} 5 \alpha$ using standard techniques in Sambrook et al. (1989) and induced for expression using $1 \mathrm{mM}$ isopropyl $\beta$-D-1thiogalactopyranoside. At each cloning step, five clones were sequenced to identify clones with correct inserts. The activity of expressed $\operatorname{lux} S_{72}$ in $E$. coli $\mathrm{DH} 5 \alpha$ was measured using the TL-26 AI-2 reporter as described above; and E. coli M4100, which can produce AI-2 (Sperandio et al., 1999), was used as positive control.

Clone library construction of luxS genes amplified from sponge-associated bacterial communities

Degenerate primers VluxsF and VluxsR were used to PCR-amplify putative Vibrio luxS genes from total genomic DNA extracted from sponges M. laxissima and I. strobilina, and also from surrounding seawater using the same PCR conditions used to amplify luxS genes from bacterial isolates. The PCR products were gel purified using a QIAquick gel extraction kit (Qiagen). Corresponding areas from the negative control sample in which the PCR was performed with no added template DNA were excised and taken through the same cloning procedure to provide strict negative controls. Purified PCR products were ligated into the pCR-XL-TOPO vector and transformed into One Shot TOP 10 chemically competent E. coli cells using a TOPO XL PCR cloning kit (Invitrogen Life Technologies). Plasmid DNA was isolated from individual clones and purified using a SprintPrep 384 kit (Agencourt Bioscience, Beverly, MA, USA). Sequencing was performed as described using M13 forward sequencing primer.

Phylogenetic analysis of $16 S$ rRNA gene sequences and luxS gene sequences

16S rRNA gene sequences from isolates were edited using PreGap3 and Gap4 from the Staden package and analyzed using BLASTn at the NCBI website. Isolates were presumptively identified according to the identity of the closest cultured relative. Partial 16S rRNA gene sequences of all the Vibrio isolates were aligned using Clustal $\times 2.0 .12$ (http://www. clustal.org/) and a phylogenetic tree was constructed using software MEGA 4.0 (http://www.megasoft ware.net/). luxS gene sequences from all the Vibrio isolates were converted to amino acid (aa) sequence using open reading frame finder and BLASTx at the NCBI website. luxS gene sequences from clone libraries were edited and the vector sequences were removed using the VecScreen tool at the NCBI website and were converted into aa sequences as described for isolates. The luxS gene sequences from isolates and clones were aligned and the phylogenetic tree was constructed as described for the $16 \mathrm{~S}$ rRNA gene. To examine the novelty of the new cluster of isolates with $\operatorname{luxS}$ genes obtained in this study, the novel luxS genes were analyzed using BLASTn and the top 100 hits in Genbank were obtained. In addition, luxS genes in Vibrio spp. were searched for in Genbank using the key words 'Vibrio AND luxS'. These two data sets were pooled and aligned together with all the $\operatorname{luxS}$ sequences obtained from this study. In order to present a comprehensive but legible tree, the following approach was used: if the reference sequences from GenBank shared higher than 99\% similarity with each other, then only one representative was chosen for inclusion in the tree and the total number of sequences that shared over $99 \%$ similarity to that representative was given in brackets. The name and accession number of these sequences is presented in Supplementary Table.

Rarefaction analyses of the luxS genes clone libraries were performed using the mothur program (Schloss et al., 2009).

\section{Nucleotide sequence accession numbers}

16S rRNA gene sequences from isolates were submitted to GenBank under accession no. HM117107-HM117132. luxS gene sequences from isolates were submitted to GenBank under accession no. HM117133-HM117158 and luxS gene sequences from clone libraries were submitted to GenBank under accession no HM117021-HM117106.

\section{Results}

Partial luxS sequence and detection of AI-2 activity of Vibrio isolates from sponges

In total, 14 out of 47 isolates from M. laxissima, 10 out of 40 isolates from I. strobilina and 2 out 36 isolates from surrounding seawater were identified as Vibrio spp. by $16 \mathrm{~S}$ rRNA gene sequences analysis ( $>1300 \mathrm{bp}$ ). For M. laxissima, the plate count was $1.2 \pm 0.2 \times 10^{7}$ colony-forming units (c.f.u.) $\mathrm{cm}^{-3}$ of sponge tissue (mean \pm s.e.), for I. strobilina, the plate count was $2.6 \pm 0.8 \times 10^{7}$ c.f.u. $\mathrm{cm}^{-3}$ of sponge tissue and for surrounding seawater, the plate count $2.0 \pm 0.5 \times 10^{5}$ c.f.u. $\mathrm{ml}^{-1}$. Total Vibrio isolates in $1 \mathrm{~cm}^{3}$ sponge tissue were calculated at $\mathrm{ca}$ $3.5 \times 10^{6}$ c.f.u. and $6.5 \times 10^{6}$ c.f.u. for M. laxissima and I. strobilina, respectively. For surrounding seawater, the culturable Vibrio count was ca $1.1 \times 10^{4}$ c.f.u. $\mathrm{ml}^{-1}$. Total bacterial counts obtained by microscopic enumeration of 4,6-diamidino-2phenylindole-stained cells was $1.1 \pm 4.4 \times 10^{9}$ cells cm ${ }^{-3}$ and $2.8 \pm 0.4 \times 10^{9}$ cells $\mathrm{cm}^{-3}$ for $M$. laxissima and I. strobilina, respectively (Mohamed, 2007; Mohamed et al., 2008d). 


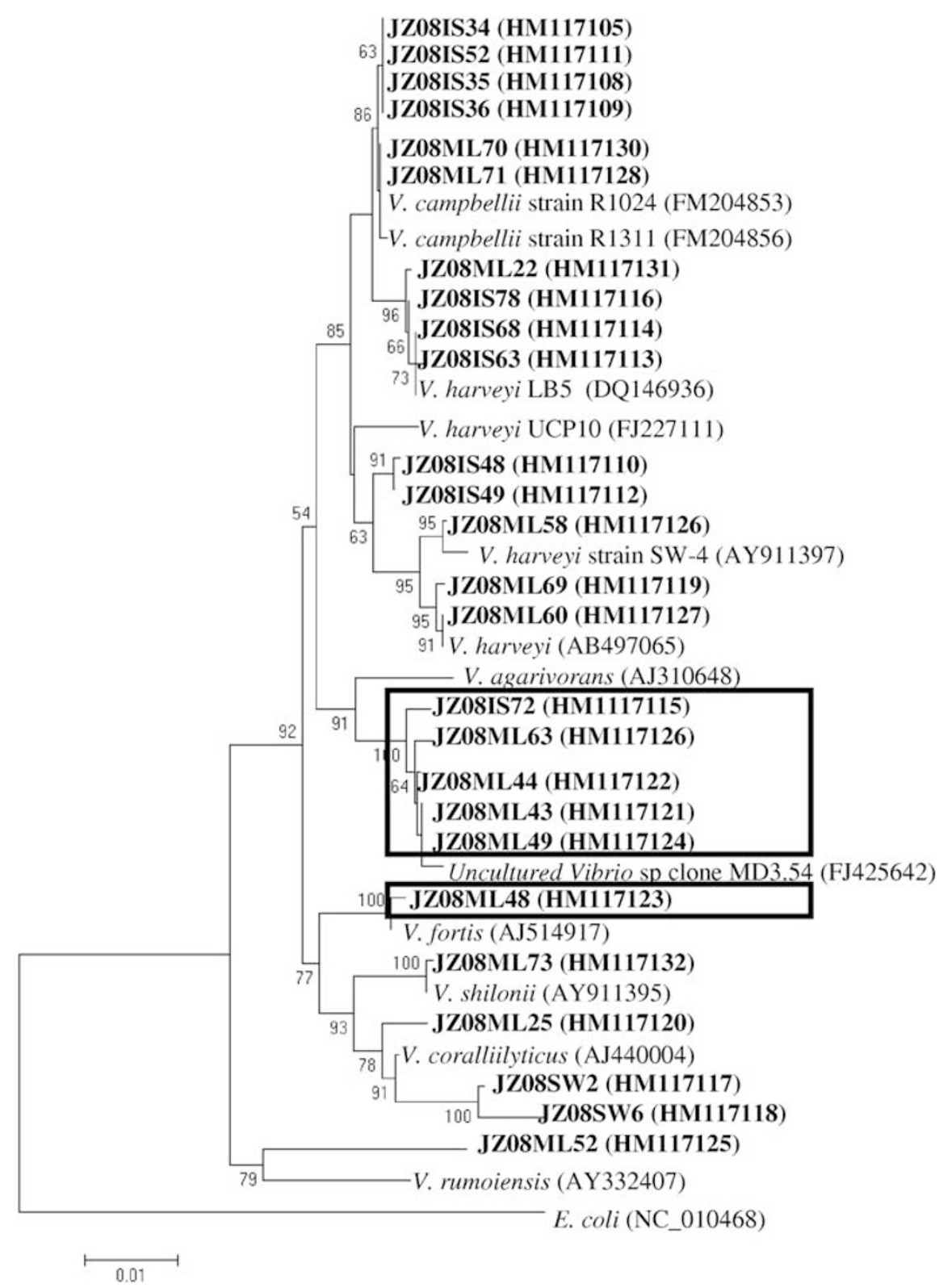

Figure 1 Rooted neighbor-joining phylogenetic tree of partial 16S rRNA gene sequences ( $>1300$ bp) of Vibrio strains that were recovered from M. laxissima (JZ08ML), I. strobilina (JZ08IS) and seawater (JZ08SW). All the sequences retrieved in this study are in bold and accession numbers are listed in parentheses for each entry. Isolates in box represent the Vibrio isolates that carry novel luxS gene sequences shown in Figure 2. Reference sequences are shown with GenBank accession numbers listed after each sequence name. Bootstrap values $>50 \%$ are shown at nodes. The scale bar indicates 0.10 substitutions per nucleotide position.

As shown in the phylogenetic tree (Figure 1), a variety of different Vibrio species were retrieved. Six and nine isolates from the two sponges were closely related to strains of $V$. campbelli and $V$. harveyi, respectively. The other isolates from sponges were closely related to various Vibrio species, such as Vibrio agarivorans, Vibrio shilonii and Vibrio fortis. JZ08ML52 shared only 96\% identity to its closest relative Vibrio rumoiensis (AY332407), indicating this strain might be a new species. 16S rRNA genes of two isolates from seawater (JZ08SW2 and JZ08SW6) shared 96-97\% identity to that of
Vibrio coralliilyticus (DJ440004), indicating that these isolates may also be new Vibrio spp.

Degenerate primers VluxsF and VluxR based on Vibrio luxS sequences were able to amplify partial luxS gene sequences from all the Vibrio isolates. A phylogenetic tree of the $\operatorname{lux} S$ gene sequences from these vibrios is shown in Figure 2. Using the approach described above to examine the novelty of the luxS cluster MI, about 130 sequences were analyzed. The tree based on Vibrio luxS sequences only (Figure 2) was very similar to that obtained by including all luxS sequences (Supplementary 


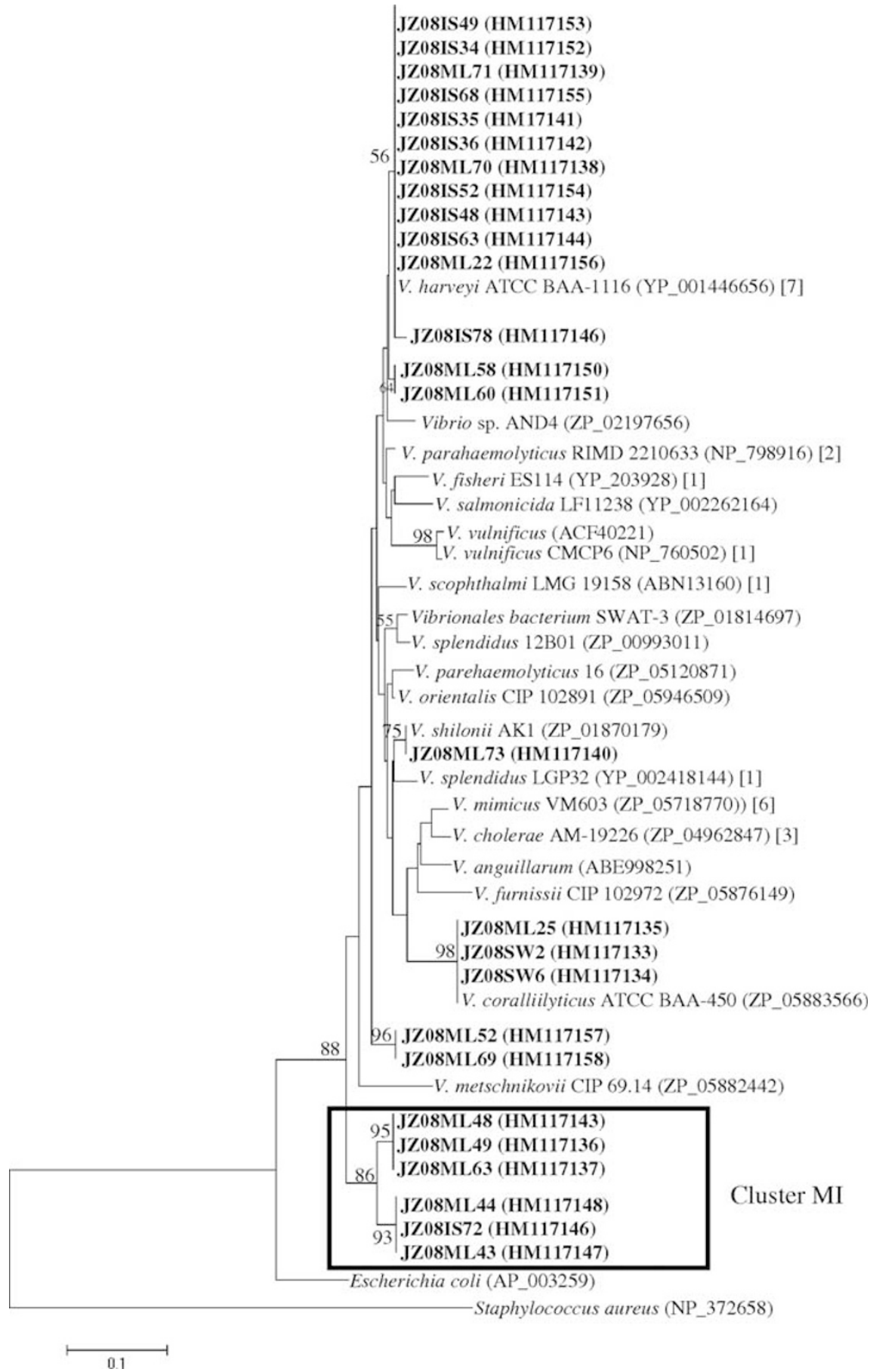

Figure 2 Phylogenetic tree using neighbor-joining method based on the predicted 96 aa residues encoded by luxS genes from Vibrio isolates. Sequences isolated from M. laxissima (JZ08ML), I. strobilina (JZ08IS) and seawater (JZ08SW) in this study are in bold and the accession number of each of these luxS sequences is listed after the isolate name. The numbers in [] indicates the numbers of sequences in Genbank that shared over 99\% identity to the sequence on the tree and these sequences are listed in Supplementary Material Table 1. The novel luxS cluster is named Cluster MI. Bootstrap values $>50 \%$ is shown at nodes. The scale indicates the number of aa substitutions per site.

Figure 1). The majority of luxS genes from these sponge-derived Vibrio isolates are closely related to that of $V$. harveyi, which is consistent with the close relationship between these isolates and $V$. harveyi shown by $16 \mathrm{~S}$ rRNA phylogeney. However, in several cases the phylogeny of luxS genes does not match that indicated by the corresponding $16 \mathrm{~S}$ rRNA genes. For example, the $16 \mathrm{~S}$ rRNA gene sequences of isolates JZ08ML69 and JZ08ML52 are closely related to those of $V$. harveyi (AB497065) and $V$. rumoiensis (AY332407), respectively; but the luxS gene sequences of these two isolated shared over 


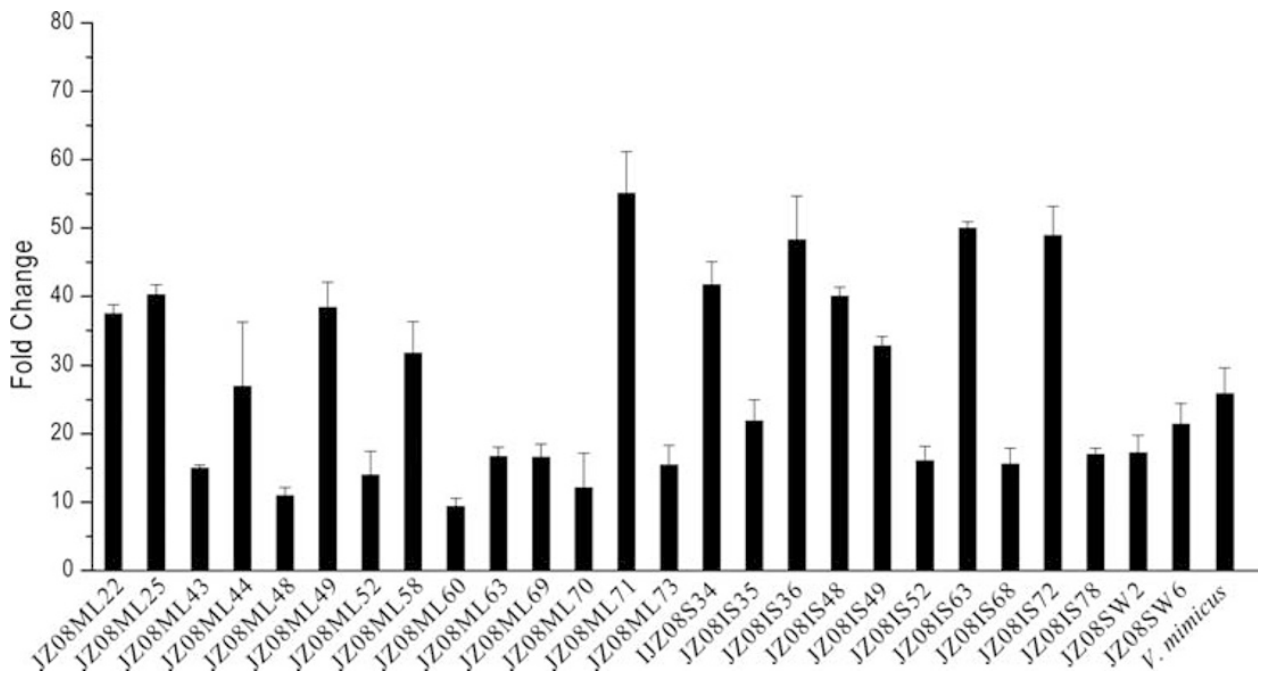

Figure 3 AI-2 activities of all Vibrio isolates measured by reporter strain $V$. harveyi TL-26. Fold changes were calculated by comparing the light production induced by supernatant of individual log phase cultures to that using Luria-Bertani (LB) medium instead of culture supernatant. V. mimicus ATCC 33653 was used as positive control. Supernatant from A. tumefaciens KYC55 failed to induce light production. Bars are means from triplicate experiments; error bars represented the s.e.m.

99\% identity to each other. Similarly, the $16 \mathrm{~S}$ rRNA genes of isolate JZ08ML48 grouped with $V$. fortis (AJ514917), but its luxS gene sequence clustered with the luxS genes from isolates JZ08ML43, JZ08ML44, JZ08ML49, JZ08ML63 and JZ08IS73.

A novel cluster (named cluster MI) of luxS gene sequences, comprising those from isolates JZ08ML43, JZ08ML44, JZ08ML48, JZ08ML49, JZ08ML63, JZ08IS73, shared only 88-90\% identity to their closest relative in Genbank (boxed in Figure 2). JZ08IS72 was chosen as a representative of this group for further analysis to confirm the novelty of these luxS genes.

All the isolates are evaluated for AI-2 activity using reporter strain $V$. harveyi TL-26. All isolates were able to induce light production (Figure 3) whereas A. tumefaciens KYC55 did not induce light production (data not shown), showing that all the Vibrio isolates, all of which have luxS genes, are able to synthesize AI-2-type molecules.

JZ08IS72 was grown in shaken culture in LuriaBertani medium with $2 \% \mathrm{NaCl}$ at $30^{\circ} \mathrm{C}$. AI-2 activity in the culture supernatant was detected from early exponential phase $(2 \mathrm{~h}$ after inoculation $)$ and onward. It reached the maximal level during transition to stationary phase (6 h after inoculation) and decreased afterward with some fluctuations as shown in Figure 4.

Characterization of luxS sequence of JZ08IS72 and expression in E. coli $\mathrm{DH} 5 \alpha$

Using a genome walking method, the full length of $\operatorname{lux} S_{72}$ was cloned. Results showed that an open reading frame encoding a putative luxS gene designated $\operatorname{lux} S_{72}$ had 516 nucleotides and encoded 171 aa. It shared $88 \%$ identity with its closest luxS

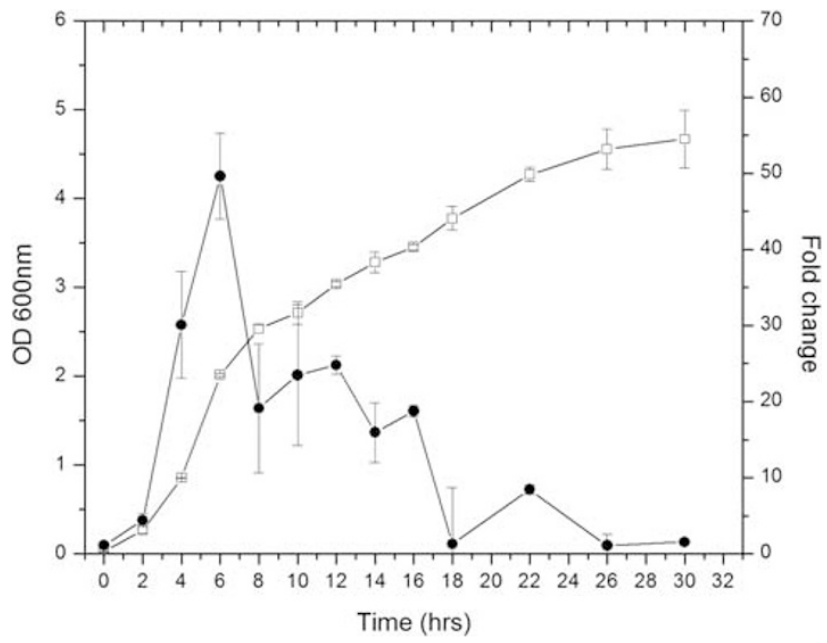

Figure 4 AI-2 production profile during growth of strain JZ08IS72. Strain JZ08IS72 was grown in Luria-Bertani (LB) with $2 \% \mathrm{NaCl}$. The growth (open squares) and AI-2 activity (closed circles) were monitored at the indicated time intervals. The error bars represent s.e. of triplicate samples.

relative from Vibrio parahaemolyticus 16 (accession number ZP_05120871) at the aa level, although no significant similarity was found between $\operatorname{lux} S_{72}$ and that of $V$. parahaemolyticus 16 at the nucleotide level, indicating high divergence of DNA sequence of $\operatorname{lux} S_{72}$. Alignment of $\operatorname{lux} S_{72}$ to that of several other Vibrio species indicated that the variance of aa largely occurred in the $C$-terminus of the protein and $\operatorname{lux} S_{72}$ has all the conserved aa identified in Hilgers and Ludwig (2001) (see Supplementary Material Figure S2).

By walking upstream and downstream of $\operatorname{lux} S_{72}$ gene, partial sequence of the glutamate-cysteine ligase gene (closely related to that of $V$. harveyi 
1512

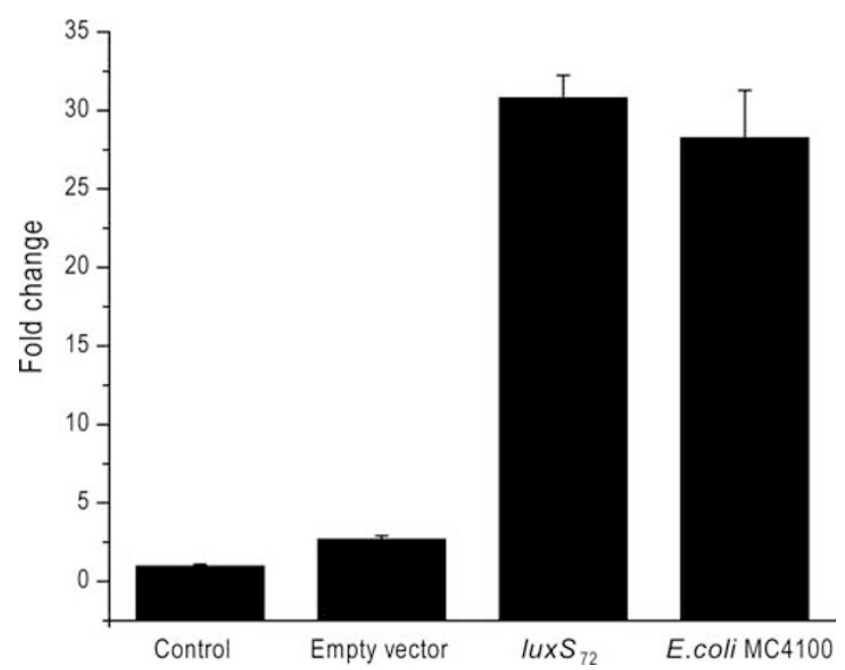

Figure 5 Heterologous expression of $\operatorname{luxS}_{72}$ in E. coli DH5 $\alpha$. $\operatorname{luxS}_{72}$ was cloned into expression vector pSRK-Gm and chemically transformed into E. coli DH5 $\alpha$. The transformant was grown in Luria-Bertani (LB) at $37^{\circ} \mathrm{C}$ up to an $\mathrm{OD}_{600}$ of $c a 1.2$ and induced by isopropyl $\beta$-D-1-thiogalactopyranoside $(1 \mathrm{mM})$ for $3 \mathrm{~h}$. E. coli MC4100 was used as a positive control. All the strains were grown up to an $\mathrm{OD}_{600}$ of $1.80 \pm 0.02$. The AI- 2 activity was measured as described in Materials and methods section. The bars (from the left) represent the result of AI-2 activity from E. coli $\mathrm{DH} 5 \alpha$, the empty vector control, E. coli DH5 $\alpha$ expressing $\operatorname{lux} S_{72}$, and the positive control E. coli MC4100. Experiments were performed in triplicate and error bars indicate the s.e.m.

HY01 (ZP_01988480)) was found upstream of $\operatorname{lux} S_{72}$ and partial sequence of a putative $\mathrm{Mg}^{2+}$ and $\mathrm{Co}^{2+}$ transporter $\operatorname{cor} B$ gene was found downstream of $\operatorname{lux} S_{72}$ (data not shown).

In order to confirm the function of the novel $\operatorname{luxS}_{72}$, it was cloned into the pSRKGm expression vector, placing it under control of the isopropyl $\beta$-D-1thiogalactopyranoside-inducible $\operatorname{lac} Z$ promoter $\left(P_{\mathrm{Lac}}\right)$ and expressed in E. coli $\mathrm{DH} 5 \alpha$, which does not produce AI-2 because of a mutation in the luxS gene (Surette and Bassler, 1998). Results shown in Figure 5 demonstrate that $\operatorname{lux} S_{72}$ was successfully expressed in the heterologous host. Significant AI-2 activity was detected compared with the empty vector and control $(P<0.05)$ and the heterologous production of AI-2 because of expression of lux $S_{72}$ was comparable to a luxS-proficient strain E. coli MC4100 $(P>0.05)$.

Sequence analysis of luxS genes from clone libraries luxS genes were successfully PCR-amplified from marine sponges M. laxissima and I. strobilina and also from surrounding seawater from Key Largo, using degenerate primers VluxSF and VluxSR. Phylogenetic relationships of the luxS-deduced aa sequences from these two marine sponges and surrounding seawater samples were analyzed. Phylogenetic relationships based on nucleotide sequences were also determined. Overall, these two trees based on aa sequences and nucleotide sequences were generally congruent with each other (data not shown). In total, 33, 30 and 23 clones of luxS genes were recovered and sequenced from the sponges M. laxissima, I. strobilina and seawater, respectively. To determine the relative richness and coverage of $\operatorname{luxS}$ genes sequenced from M. laxissi$m a$, I. strobilina and surrounding seawater, rarefaction analyses were performed. The rarefaction curves for luxS genes from $M$. laxissima and I. strobilina approached a plateau, and nearly plateaued for surrounding seawater at the distance of 0.01 (see Supplementary Material Figure S3). At the distance of 0.03 , all the three rarefaction curves reached a plateau, indicating sufficient sampling of these clone libraries (data not shown). The phylogenetic analysis of these sequences is shown in Figure 6. In all, 31 out of 33 clones from $M$. laxissima, referred to as ML group 1 shared 97-99\% identity to luxS gene of $V$. mimicus VM603 (ZP_05718770), and the other two clones, referred to as ML group 4 shared $100 \%$ identity to the luxS gene sequence of $V$. harveyi ATCC BAA-1116 (YP_001446656). In total, 28 out of 30 clones from I. strobilina and nine clones from seawater, referred to as IS and SW group 5, were closely related to the luxS gene of V. harveyi 1DA3 (ZP_06175640), sharing 96-99\% identity and the other two clones from I. strobilina (IS group 3) shared 94\% identity to the luxS gene of $V$. parahaemolyticus RIMD 2210633 (NP_798916). In all, 14 out of 23 clones from seawater, referred to as the SW group 2, were closely related to the luxS gene of $V$. mimicus VM603 (ZP_05718770), sharing 98-99\% identity.

\section{Discussion}

The marine sponges M. laxissima and I. strobilina contained several different Vibrio strains, identified by $16 \mathrm{~S}$ rRNA gene sequence analysis. Associations of vibrios with other marine organisms range from pathogenic to mutualistic. Close relatives of the Vibrio isolates described in this study include $V$. harveyi, a major pathogen of marine animals (Zhang et al., 2001) and V. shiloli (AF007115), well established as a causative agent of coral bleaching (Kushmaro et al., 1996, 1997). Pathogenicity is often strain-specific rather than species-specific so the close relationship between the sponge-associated Vibrio species and known pathogens does not necessarily indicate that our Vibrio isolates are potential pathogens of their host sponges. Known mutualist relationships between Vibrio spp. and marine invertebrates include Vibrio fischeri-bobtail squid (McFall-Ngai, 2008). Sponge-associated vibrios might offer the host sponge or the associated bacterial communities some benefits. One possibility is that nitrogen-fixing vibrios may supply the holobionts with fixed nitrogen. Shieh and Lin (1994) described vibrios as nitrogen-fixing bacteria associated with sponge Halichondria. However, none of the vibrios isolated in this study are closely related 


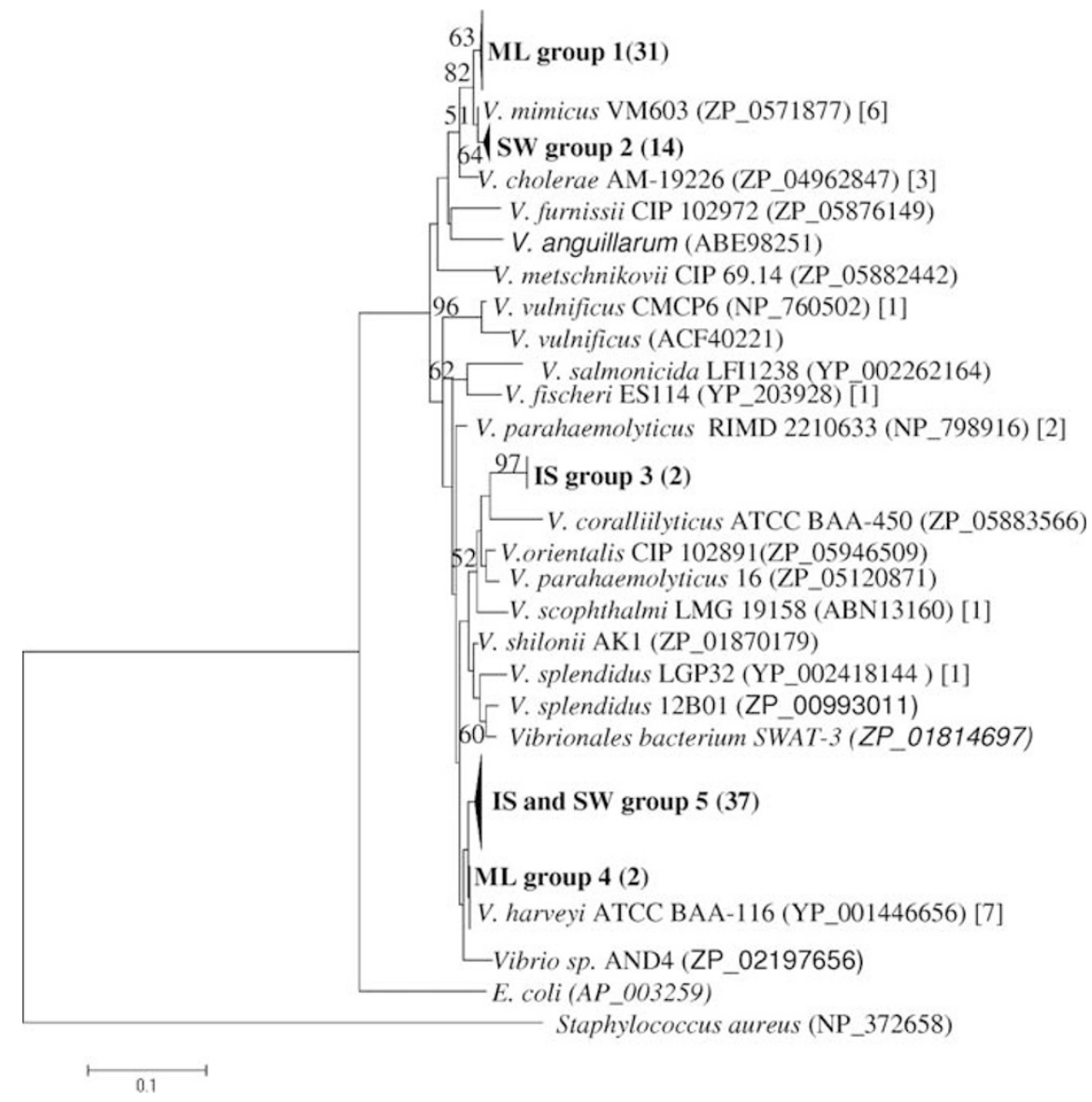

Figure 6 Phylogenetic tree using the neighbor-joining method based on the predicted 117 aa residues encoded by the luxS gene amplified from the bacterial communities of M. laxissima (ML), I. strobilina (IS) and surrounding seawater (SW). Sequences obtained in this study are in bold. Bootstrap values $>50 \%$ are shown at each node. The scale bar indicates the number of aa substitutions per site. Numbers in () after each group name indicate the number of clones in each group. See Supplementary Material Table 1 for accession numbers of each clone. Numbers in [] after reference sequences are the same as in Figure 2 legend. See Supplementary Material Table 2 for accession numbers of each clone.

to previously described nitrogen-fixing Vibrio spp. and none of the nifH gene sequences retrieved from the microbial communities of M. laxissima and I. strobilina is closely related to the nifH genes of vibrios (Mohamed et al., 2008b). The role of the Vibrio spp. associated with $M$. laxissima and I. strobilina therefore remains speculative.

Using degenerate luxS primers based on Vibrio $\operatorname{lux} S$ gene sequences, partial luxS gene sequences were detected in all Vibrio isolates from the two marine sponges and surrounding seawater. A novel cluster containing luxS genes from six isolates shared only low identity (88-90\%) to known luxS gene sequences. Our results thus extend the known diversity of this QS gene. IuxS genes are highly divergent among gram-negative and gram-positive bacteria but might have similar functions. For example, the luxS gene of Bacillus subtilis shares only $\mathrm{Ca} 40 \%$ identity to that of $V$. cholerae and $V$. harveyi; however, AI-2 produced in B. subtilis can induce the bioluminescence of $V$. harveyi BB170 and LuxS-dependent QS is required to form a differentiated biofilm and also for swarming on solid surfaces (Lombardia et al., 2006). Further, even among the vibrios, the luxS gene of $V$. cholerae shares only $87 \%$ identity to that of $V$. harveyi yet the lux operon of $V$. harveyi can function well in $V$. cholerae (Lenz et al., 2004). This suggests that the novel luxS cluster obtained in this study might be involved in the same pathway as that of $V$. harveyi or $V$. cholerae. The $\mathrm{LuxS}_{72}$ aa sequence has all of the uniformly conserved residues for other $S$-ribosylhomocysteinases, including those implicated in metal binding (Hilgers and Ludwig, 2001). It is therefore not surprising that this protein can function to drive synthesis of AI-2 when expressed in E. coli $\mathrm{DH} 5 \alpha$.

A comparison of the plate counts obtained on marine agar and the total cell counts present in the sponges revealed that $c a 1 \%$ of sponge-associated bacteria were culturable from both sponges. Vibrios comprised a major group of bacteria in the culturable assemblage from both sponges ( $\mathrm{ca} 30 \%$ and $25 \%$ for M. laxissima and I. strobilina, respectively). We therefore focused on Vibrio spp. for this investigation of the presence of luxS genes in 
1514

sponge-associated bacteria because of the high incidence of Vibrio spp. in the total bacterial assemblage and because there is a precedent for luxS signaling to be important in colonization of a marine invertebrate by a Vibrio sp., in the V. fischeri/ Euprymna scolopes symbiosis (Lupp and Ruby, 2004). We acknowledge that the proportion of Vibrio spp. in the total sponge-associated bacterial community may be low (our data establish a lower limit of ca 3 Vibrio cells/10000 prokaryote cells in these two sponges) and that the ecological significance of luxS signaling in these communities remains to be established. A recent study obtained 25 Vibrio isolates from healthy and diseased corals and showed that all the Vibrio isolates were able to activate an AI-2 reporter, although no luxS genes were amplified in this study (Tait et al., 2010). The investigators speculated that vibrios might use several different types of QS signal molecules to regulate processes that are used to colonize corals during adverse environmental conditions. Studies by Bansal et al. (2008) and Englert et al. (2009) showed that E. coli cells are attracted by AI-2 and AI-2 can be as effective an attractant as L-aspartate. On the basis of these studies, one hypothesis is that E. coli might use AI-2 to join groups of bacteria that produce AI-2 (Defoirdt, 2010). It is also possible that chemotaxis to AI-2 might serve to attract free-living bacteria to biofilms (Hegde et al., 2011). AI-2 is better understood in Vibrios than in other bacteria. AI-2 has been well characterized in $V$. harveyi and $V$. cholerae as a signaling molecule. It controls bioluminescence, metalloprotease production and type III secretion in V. harveyi and represses biofilm and virulence and activates protease activity in V. cholerae ( $\mathrm{Ng}$ and Bassler, 2009). In light of these observations, it is possible that similar functions are controlled by AI-2 signaling in sponge-associated Vibrios.

Interestingly, in some cases there was a large difference in the levels of AI-2 activity between closely related isolates (for example, isolates JZ08ML70 and JZ08ML71 show around fivefold difference in AI-2 activity. The luxS gene has been showed to be regulated both at the transcriptional and translational levels (Zhang et al., 2008; Udekwu, 2010). The difference in the levels of AI-2 activity between closely related isolates, (for example, JZ08ML70 and JZ08ML71 show around fivefold difference in AI-2 activity) could be due to the difference in the gene regulation.

AI-2/luxS QS systems among vibrios can regulate genes involved in biofilm formation, virulence factors, bioluminescence and colonization $(\mathrm{Ng}$ and Bassler, 2009). In the well-characterized V. fischeriE. scolopes colonization system, double mutants of luxS and ains (an AHL synthase gene) reduces the already diminished level of colonization of ainS mutants by $50 \%$ (Lupp and Ruby, 2004). Almost all the Vibrio isolates obtained in this study (Zan, Fuqua and Hill, in preparation) and in previous report by Mohamed et al. (2008a) also produce AHL. The role of the AI-2/luxS system in the colonization of sponges by vibrios provides an interesting area for future work.

The AI-2 activity of JZ08IS72 peaked $6 \mathrm{~h}$ after inoculation, decreased during the transition to stationary phase and there was almost no AI-2 activity detectable after $18 \mathrm{~h}$ following inoculation. In contrast, the AI-2 activity of $V$. mimicus ATCC 33653 detected in parallel with that of JZ08IS72 did not show this pattern. In $V$. mimicus ATCC 33653, AI-2 activity peaked between 8 and $10 \mathrm{~h}$ after inoculation and remained relatively constant with some fluctuations thereafter (data not shown). There are no other reports of AI-2 systems from Vibrio species showing a peak in production followed by a decline, not even in $V$. harveyi and $V$. cholerae, in which the AI-2 QS pathway is well studied. However, in Salmonella enterica serovar Typhimurium and E. coli K-12 strain MG1655, AI-2 production peaks in mid-to-late exponential phase and quickly declines either in the transition to stationary phase or in stationary phase (Xavier and Bassler, 2005; Doherty et al., 2006). This is because of the internalization of AI-2 by the Lsr transporter in $S$. enterica serovar Typhimurium and by the b1513 transporter (a homolog of the Lsr transporter) in E. coli K-12 strain MG1655 (Xavier and Bassler, 2005). No homolog of the Lsr transporter was found in the genomes of $V$. harveyi and $V$. cholerae (data not shown). This suggests that a potential new pathway involving in internalization of AI-2 or perhaps degradation may exist in JZ08IS72, which warrants further investigation. It is also possible that other environmental factors might affect the AI-2 activity of JZ08IS72 during stationary phase, such as the lack of a preferred carbon source, neutral $\mathrm{pH}$, and low osmolarity (Surette et al., 1999). The ecological relevance of the peak-shaped pattern is not clear.

In the luxS gene clone library from M. laxissima, ML group 1 was clearly distinct from SW group 2 with a high bootstrap value of $82 \%$ and did not cluster with any other known luxS gene sequences, implying that ML group 1 might be a sponge-specific $\operatorname{luxS}$ gene group. IS group 3 is a relatively small group that is distantly related to any known Vibrio luxS sequence, suggesting that this group comprises a sponge-specific luxS gene group. Clones from I. strobilina and seawater in IS and SW group 5 were closely related to each other, suggesting that strains carrying these genes may be derived from the surrounding seawater.

\section{Conclusions}

All the Vibrio spp isolated from two marine sponges were shown to have luxS gene and were demonstrated to induce light production in a reporter strain. A novel cluster of luxS gene (cluster MI) sequences was obtained. The relationship between 
AI-2/luxS QS and the colonization on sponge hosts by vibrios deserves further investigation. This work provides the first information about luxS genes and AI-2 activity in sponge-associated bacterial communities.

\section{Acknowledgements}

We acknowledge the National Undersea Research Center (NURC), University of North Carolina at Wilmington for providing sampling opportunities in Key Largo, FL, USA. Funding for this research was provided by the National Science Foundation Directorate for Biological Sciences Molecular and Cellular Biosciences (MCB-0238515) and Integrative Organismal Systems (IOS-0919728). We thank Tao Long and Bonnie Bassler for the gift of $V$. harveyi TL-26. Ryan Powell is thanked for assistance in preparation of figures and Leah Blasiak is thanked for comments on the paper. We thank two anonymous reviewers for thoughtful comments.

\section{References}

Bansal T, Jesudhasan P, Pillai S, Wood TK, Jayaraman A. (2008). Temporal regulation of enterohemorrhagic Escherichia coli virulence mediated by autoinducer-2. Appl Microbiol Biotech 78: 811-819.

Defoirdt T. (2010). Can bacteria actively search to join groups? ISME J 5: 569-570.

Doherty N, Holden MT, Qazi SN, Williams P, Winzer K. (2006). Functional analysis of luxS in Staphylococcus aureus reveals a role in metabolism but not quorum sensing. J Bacteriol 188: 2885-2897.

Englert DL, Manson MD, Jayaraman A. (2009). Flow- based microfluidic device for quantifying bacterial chemotaxis in stable, competing gradients. Appl Environ Microbiol 75: 4557-4564.

Enticknap JJ, Kelly M, Peraud O, Hill RT. (2006). Characterization of a culturable alphaproteobacterial symbiont common to many marine sponges and evidence for vertical transmission via sponge larvae. Appl Environ Microbiol 72: 3724-3732.

Fuqua WC, Winans SC, Greenberg EP. (1994). Quorum sensing in bacteria: the LuxR-LuxI family of cell density-responsive transcriptional regulators. J Bacteriol 176: $269-275$.

Greenberg EP, JW H, Ulitzer S. (1979). Induction of luciferase in Beneckea harveyi by other bacteria. Arch Microbiol 120: 87-91.

Hegde M, Englert DL, Schrock S, Cohn WB, Vogt C, Wood TK et al. (2011). Chemotaxis to the quorum-sensing signal AI-2 requires the chemoreceptor and the periplasmic LsrB AI-2 binding protein. J Bacteriol 193: 768-773.

Henke JM, Bassler BL. (2004a). Quorum sensing regulates type III secretion in Vibrio harveyi and Vibrio parahaemolyticus. J Bacteriol 186: 3794-3805.

Henke JM, Bassler BL. (2004b). Three parallel quorumsensing systems regulate gene expression in Vibrio harveyi. J Bacteriol 186: 6902-6914.

Hentschel F, Usher KM, Taylor MW. (2006). Marine sponges as microbial fermenters. FEMS Microbiol Ecol 55: $167-177$.
Hilgers MT, Ludwig ML. (2001). Crystal structure of the quorum-sensing protein LuxS reveals a catalytic metal site. Proc Natl Acad Sci USA 98: 11169-11174.

Hooshangi S, Bentley WE. (2008). From unicellular properties to multicellular behavior: bacteria quorum sensing circuitry and applications. Curr Opin Biotechnol 19: 550-555.

Khan RS, Gaines J, Martin Roop II R, Farrand SK. (2008). Broad-host-range expression vectors with tightly regulated promoters and their use to examine the influence of TraR and TraM expression on Ti plasmid quorum sensing. Appl Environ Microbiol 74: 5053-5062.

Kushmaro A, Loya Y, Fine M, Rosenberg E. (1996). Bacterial infection and coral bleaching. Nature 380: 396.

Kushmaro A, Rosenberg E, Fine M, Loya Y. (1997). Bleaching of the coral Oculina patagonica by Vibrio AK-1. Mar Ecol Prog Ser 147: 159-165.

Lenz DH, Mok KC, Lilley BN, Kulkarni RV, Wingreen NS, Bassler BL. (2004). The small RNA chaperone Hfq and multiple small RNAs control quorum sensing in Vibrio harveyi and Vibrio cholerae. Cell 118: 69-82.

Lombardia E, Rovetto A, Arabolaza A, Grau R. (2006). A LuxS-dependent cell -to -cell language regulates social behavior and development in Bacillus subtilis. J Bacteriol 188: 4442-4452.

Lupp C, Ruby EG. (2004). Vibrio fischeri LuxS and AinS: comparative study of two signal synthases. J Bacteriol 186: $3873-3881$

Maidak BL, Cole JR, Lilburn TG, Parker CT, Saxman PR, Farris RJ et al. (2001). The RDP-II (Ribosomal Database Project). Nucleic Acids Res 29: 173-174.

McFall-Ngai M. (2008). Host-microbe symbiosis: the squid-Vibrio association-a naturally occurring, experimental model of animal/bacterial partnerships. Adv Exp Med Biol 635: 102-112.

Miller MB, Skorupski K, Lenz DH, Taylor RK, Bassler BL. (2002). Parallel quorum sensing systems converge to regulate virulence in Vibrio cholerae. Cell 110: 303-314.

Mohamed NM. (2007). Ecophysiology of Microbial Communities Associated with Marine Sponges Ircinia Strobilina and Mycale Laxissima. Ph.D. Dissertation. University of Maryland, MD, USA.

Mohamed NM, Cicirelli EM, Kan J, Chen F, Fuqua C, Hill RT. (2008a). Diversity and quorum-sensing signal production of Proteobacteria associated with marine sponges. Environ Microbiol 10: 75-86.

Mohamed NM, Colman AS, Tal Y, Hill RT. (2008b). Diversity and expression of nitrogen fixation genes in bacterial symbionts of marine sponges. Environ Microbiol 10: 2910-2921.

Mohamed NM, Enticknap JJ, Lohr JE, McIntosh SM, Hill RT. (2008c). Changes in bacterial communities of the marine sponge Mycale laxissima on transfer into aquaculture. Appl Environ Microbiol 74: 1209-1222.

Mohamed NM, Rao V, Hamann MT, Kelly M, Hill RT. (2008d). Monitoring bacterial diversity of the marine sponge Ircinia strobilina upon transfer into aquaculture. Appl Environ Microbiol 74: 4133-4143.

Mohamed NM, Saito K, Tal Y, Hill RT. (2010). Diversity of aerobic and anaerobic ammonia-oxidizing bacteria in marine sponges. ISME J 4: 38-48.

Murray MG, Thompson WF. (1980). Rapid isolation of high molecular weight plant DNA. Nucl Acids Res 8: 4321-4325.

Ng WL, Bassler BL. (2009). Bacterial quorum-sensing network architectures. Annu Rev Genet 43: 197-222. 
Pitcher DG, Saunders NA, Owen RJ. (1989). Rapid extraction of bacterial genomic DNA with guanidium thiocyanate. Lett Appl Microbiol 8: 151-156.

Rezzonico F, Duffy B. (2008). Lack of genomic evidence of AI-2 receptors suggests a non-quorum sensing role for luxS in most bacteria. BMC Microbiol 8: 154-173.

Rose TM, Henikoff JG, Henikoff SN. (2003). CODEHOP (COnsensus-DEgenerate Hybrid Oligonucleotide Primer) PCR primer design. Nucl Acids Res 31: 3763-3766.

Sambrook J, Fritsch EF, Maniatis T. (1989). Molecular Cloning: A Laboratory Manual, 2nd edn Cold Spring Harbor Laboratory Press: Cold Sponge Harbor, NY.

Schloss PD, Westcott SL, Ryabin T, Hall JR, Hartmann M, Hollister EB et al. (2009). Introducing mother: opensource, platform-independent, community-supported software for describing and comparing microbial communities. Appl Environ Microbiol 75: 7537-7541.

Shieh W, Lin Y. (1994). Association of heterotrophic nitrogen-fixing bacteria with a marine sponge of Halichondria sp. Bull Mar Sci 54: 557-564.

Somerville CC, Knight IT, Straube WL, Colwell RR. (1989). Simple, rapid method for direct isolation of nucleic acids from aquatic environments. Appl Environ Microbiol 55: 548-554.

Sperandio V, Mellies J, Nguyen W, Shin S, Kaper J. (1999). Quorum sensing controls expression of the type III secretion gene transcription and protein secretion in enterohemorrhagic and enteropathogenic Escherichia coli. Proc Natl Acad Sci USA 96: 15196-15201.

Surette M, Bassler B. (1998). Quorum sensing in Escherichia coli and Salmonella typhimurium. Proc Natl Acad Sci USA 95: 7046-7050.

Surette MG, Miller MB, Bassler BL. (1999). Quorum sensing in Escherichia coli, Salmonella typhimurium, and Vibrio harveyi: a new family of genes responsible for autoinducer production. Proc Natl Acad Sci USA 96: 1639-1644.

Tait K, Hutchison Z, Thompson F, Munn CB. (2010). Quorum sensing signal production and inhibition by coral-associated vibrios. Environ Microbiol Rep 2: 145-150.

Taylor MW, Radax R, Steger D, Wagner M. (2007). Spongeassociated microorganisms: evolution, ecology, and biotechnological potential. Microbiol Mol Biol Rev 71: 295-347.
Taylor MW, Schupp PJ, Baillie HJ, Charlton TS, Nys RD, Kjelleberg S et al. (2004). Evidence for acyl homeserine lactone signal production in bacteria associated with marine sponges. Appl Environ Microbiol 70: 4387-4389.

Tiaden A, Spirig T, Hilbi H. (2010). Bacterial gene regulation by $\alpha$-hydroxyketone signaling. Trends Microbiol 18: 288-297.

Thacker RW. (2005). Impacts of shading on spongecyanobacteria symbioses: a comparison between host-specific and generalist associations. Integr Comp Biol 45: 369-376.

Udekwu KI. (2010). Transcriptional and post-transcriptional regulation of the Escherichia coli luxS mRNA; involvement of the sRNA MicA. PLoS ONE 5: e13449.

Vacelet J. (1975). Etude en microscopie electronique de l'association enter bacteries et spongiaires du genre Verongia (Dictyoceratida). J Microscopie de Biologie Cellulaire 23: 271-288.

Vacelet J, Donadey C. (1977). Electron microscope study of the association between some sponges and bacteria. $J$ Exp Mar Biol Ecol 30: 301-314.

Webster NS, Blackall LL. (2009). What do we really know about sponge-microbial symbioses? ISME J 3: 1-3.

Wilkinson CR. (1978). Microbial associations in sponges. II. Numerical analysis of sponge and water bacterial populations. Mar Biol 49: 169-176.

Wilkinson CR. (1979). Bdellovibrio-like parasite of cyanobacteria symbiotic in marine sponges. Arch Microbiol 123: 101-103.

Xavier KB, Bassler BL. (2005). Regulation of uptake and processing of the quorum-sensing autoinducer AI-2 in Escherichia coli. J Bacteriol 187: 238-248.

Zhang M, Sun K, Sun L. (2008). Regulation of autoinducer 2 production and $\operatorname{lux} S$ expression in a pathogenic Edwardsiella tarda strain. Microbiology 154: 2060-2069.

Zhang XH, Meaden PG, Austin B. (2001). Duplication of hemolysin genes in a virulent isolate of Vibrio harveyi. Appl Environ Microbiol 67: 3161-3167.

Zhu J, Chai Y, Zhong Z, Li S, Winans S. (2003). Agrobacterium bioassay strain for ultrasensitive detection of $\mathrm{N}$-acylhomoserine lactone -type quorumsensing molecules: detection of autoinducers in Mesorhizobium huakuii. Appl Environ Microbiol 69: 6949-6953.

Supplementary Information accompanies the paper on The ISME Journal website (http://www.nature.com/ismej) 